\title{
Ayuda ergonómica para atenuar trastornos osteomioarticulares por levantamiento manual de cargas en el despacho de pollos pelados (c) (1) (2)(2)
}

Ergonomic help to attenuate osteomyoarticular disorders by manual lifting of loads in the dispatch of peeled chickens

\author{
Stefany Maritza Pilatásig Villamarín. ${ }^{1}$, Manolo Alexander Córdova Suárez. ${ }^{2}$ \& Edison
} Patricio Villacres Cevallos. ${ }^{3}$

Recibido: 09-07-2021 / Revisado: 02-07-2021 /Aceptado: 23-08-2021/ Publicado: 05-09-2021

\section{DOI: https://doi.org/10.33262/anatomiadigital.v4i3.1.1919}

\begin{abstract}
Introduction, the execution of handicraft activities where prevention management is not considered and fewer ergonomic aids expose workers to severe working conditions for lifting loads of different weights all day can cause osteomyoarticular disorders and even work accidents. Objective. The effect of an ergonomic aid was investigated to reduce the weight of the loads lifted by the Incubandina company's chicken drawer dispenser by half and to attenuate the composite lifting index of the National Institute for Occupational Safety
\end{abstract}

\section{Resumen}

Introducción, la ejecución de actividades artesanales donde no se considera gestión de la prevención y menos ayudas ergonómicas exponen a los trabajadores a condiciones severas de trabajo por el levantar cargas de diferentes pesos todo el día puede ocasionar trastornos osteomioarticulares y hasta accidentes laborales. Objetivo. Se investigó el efecto de una ayuda ergonómica para disminuir a la mitad el peso de las cargas que levanta el despachador de gavetas de pollos de la empresa Incubandina y atenuar el índice de

1 Regional Autonomous University of Los Andes, Postgraduate, Ambato, Ecuador, pg.stefanympv12@uniandes.edu.ec, ORCID: (D0000-0002-4930-7543

2 Universidad Nacional de Chimborazo, Facultad de Ingeniería, Riobamba-Ecuador, manolo.cordova@unach.edu.ec ORCID: iD0000-0001-6786-7926

3 Universidad Nacional de Chimborazo, Facultad de Ingeniería, Riobamba-Ecuador, pvillacres@ unach.edu.ec ORCID: (iD) 0000-0001-9518-1278 
and Health (NIOSH). Methodology. First, it began by identifying: time of execution of the lifts, weight of the load, vertical position (V), horizontal position $(\mathrm{H})$, height of the lift (D), angle of rotation (A), frequency of the lifts (F) and grip. Then the calculation of the individual index of each load and in the end the composite lifting index for multiple tasks was carried out, making the adjustments of the NIOSH factors using the NTP 477. This was done in initial conditions and then with the ergonomic help to see the percentage of improvement. Results. The lifting of four different loads was identified: $20 \mathrm{~kg}, 25 \mathrm{~kg}, 18 \mathrm{~kg}, 20 \mathrm{~kg}$; of which the indices of individual lifts were calculated determining: $1.15 ; 1.51 ; 1.24$ and 1.28 respectively and a composite lift index of 2.42. When locating a mirror post and sharing the loads, an individual lifting index of: 0.49 was found; $0.62 ; 0.46$; and 0.52 respectively and a compound lifting index of 0.6 Decreasing the composite lifting index by $182 \%$. Conclusion. With the use of a person who helps with the manual lifting of the loads, the compound lifting index can be lowered by $182 \%$, guaranteeing the execution of the activity within the limits recommended by Ecuadorian legislation in DE 2393 Art. 128 Lit. Four.

Keywords: Peeled chickens, lifting index, NIOSH, manual lifting of loads. levantamiento compuesto del National Institute for Occupational Safety and Health (NIOSH) compuesto. Metodología. Primero se empezó identificando: tiempo de ejecución de los levantamientos, peso de la carga, posición vertical $(\mathrm{V})$, posición horizontal $(\mathrm{H})$, altura del levantamiento (D), ángulo de giro (A), frecuencia de los levantamientos (F) y el agarre. Luego se efectuó el cálculo del índice individual de cada carga y al final el índice de levantamiento compuesto para múltiples tareas, realizando los arreglos de los factores del NIOSH utilizando la NTP 477. Esto se realizó en condiciones iniciales y luego con la ayuda ergonómica para ver el porcentaje de mejora. Resultados. Se identificó el levantamiento de cuatro cargas diferentes: $20 \mathrm{~kg}, 25 \mathrm{~kg}$, $18 \mathrm{~kg}, 20 \mathrm{~kg}$; de los cuales se calculó los índices de levantamientos individuales determinando: 1,$15 ; 1,51 ; 1,24$ y 1,28 respectivamente $y$ un índice de levantamiento compuesto de 2,42. Al ubicar un puesto espejo y compartir las cargas se encontró un índice de levantamiento individual de: 0,$49 ; 0,62$; 0,46; y 0,52 respectivamente y un índice de levantamiento compuesto de 0,6 Disminuyendo un $182 \%$ el índice de levantamiento compuesto. Conclusión. Con el uso de una persona que ayude al levantamiento manual de las cargas se logra bajar un $182 \%$ el índice de levantamiento compuesto garantizando la ejecución de la actividad dentro de los límites recomendados por la legislación ecuatoriana en el DE 2393 Art. 128 Lit. 4. 
Palabras claves: Pollos pelados, índice de levantamiento, NIOSH, levantamiento manual de cargas.

\section{Introducción}

Los mercados competitivos en la comercialización de carne de pollo obligan a los productores a desarrollar sus operaciones de manera rápida con escasos recursos y sin planificación (Suclli, 2018). Esto complica ergonómicamente a la mayoría de los puestos de trabajo y en especial a los trabajadores que ejecutan levantamiento manual de cargas ya que se exponen a ejecutar tareas frecuentes, levantado cargas pesadas en gavetas, sin consideraciones técnicas y menos ayudas biomecánicas o de prevención que atenúe los posibles efectos negativos en su salud con la presencia de trastornos osteomioarticulares (Tobar, 2018).

El personal de despacho maneja cargas de diferentes pesos de acuerdo con la cantidad y el tipo de cliente que solicita el producto (Mora, 2011). Esta variación de las cargas imposibilita el uso de ayudas mecánicas que son diseñadas para manejar el mismo peso. Además la exposición al frio por la necesidad del control de temperatura para mantener y preservar los pollos pelados aceleran los procesos de enfermedades ocupacionales y la presencia de manifestaciones tempranas relacionadas con trastornos osteomioarticulares (Márquez et al., 2015), siendo más recursivas la daños en miembros superiores, espalda baja y zona lumbar en general.

Estas actividades exigen movimientos rápidos, continuos y posiciones incomodas en sitios resbalosos fríos de una manera continua, que sumado a la falta de capacitación y entrenamiento complica la situación para el empleado y su productividad (Muñoz, 2019). En la actualidad las empresas utilizan la ergonomía de diseño como herramienta fundamental para atenuar la exposición a los factores determinantes en el levantamiento manual de cargas (Aguirre \& Ortiz, 2018). Pero cuando las ayudas biomecánicas o mecánicas no se pueden implementar por la diversidad de las cargas que se levanta el trabajador se puede pensar en utilizar maneras de organizar el trabajo y hasta compartir la carga con otra persona para mejorar el puesto y atenuar las molestias osteomioarticulares (Yaguari \& Fabiola, 2015).

Aunque la solución parece evidente se debe considerar que una disminución de las cargas no garantiza cumplir con el levantamiento recomendado por la legislación ecuatoriana es por eso que se debe calcular el índice de levantamiento compuesto con la ayuda de métodos aceptados por la Organización Internacional del Trabajo (OIT) (Rodríguez et al., 2010). Entre los métodos más utilizados está el uso de la ecuación de índice de levantamiento compuesto del National Institute for Occupational Safety and Health (NIOSH) (Cuixart \& Bravo, 1998). Este método que utiliza los datos del posicionamiento de la persona y su configuración 
espacial con la carga para ejecutar el levantamiento da un número que ayuda a entender si el trabajador supera o no el límite de carga establecida por el mismo autor que es de $23 \mathrm{~kg}$. Y además incorpora una metodología para calcular un índice global o compuesto cuando se ejecutan levantamientos de diversas cargas con diferente peso.

Esta investigación intenta determinar la mejora porcentual al implementar un puesto espejo en la ejecución de levantamientos de diferentes pesos de gavetas de pollos pelados en la empresa Incubandina.

\section{Metodología}

Ecuación del National Institute for Occupational Safety and Health (NIOSH), el diseño ergonómico garantiza un control de los factores principales en la ejecución de tareas que involucran exposición a tareas peligrosas en la aparición de trastornos osteomioarticulares. Estas tareas obligan al trabajador a realizar sobreesfuerzos al empujar cargas (Ruiz, 2011). El National Institute for Occupational Safety and Health (NIOSH) en 1981 generó una ecuación para evaluar el manejo manual de cargas en el trabajo. Su intención inicial fue identificar los riesgos de lumbalgias asociados a la carga física pero en el año 1991 se revisó dicha ecuación introduciendo criterios (Valois \& Rivera, 2019): a) biomecánico, b) fisiológico y c) psicofísico de tal manera que ubica al trabajador en posición y distancia con la carga que maneja, como se muestra en la figura 1.

\section{Figura 1}

Elementos principales entre Bípedo y carga para uso de NIOSH. Adaptado de software en línea www.ergonaurtas.upv.es

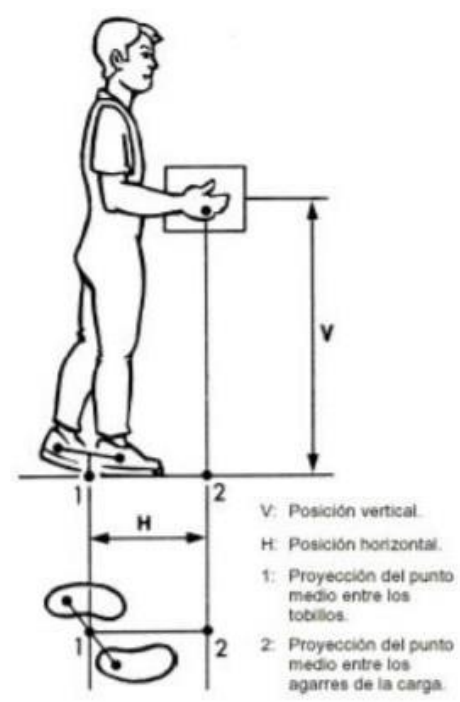


El NIOSH considera una ecuación con seis elementos principales de longitudes y una constante de carga (Ruiz, 2011). Ver ecuación 1:

$$
L P R=L C * H M * V M * D M * A M * F M * C M \quad E c u .1
$$

Donde

$\mathrm{LPR}=$ límite de peso recomendado

$\mathrm{LC}=$ constante de carga

$\mathrm{HM}=$ factor de distancia horizontal

$\mathrm{VM}=$ Factor de altura

$\mathrm{DM}=$ factor de desplazamiento vertical

$\mathrm{AM}=$ factor de asimetría

$\mathrm{FM}=$ factor de frecuencia

$\mathrm{CM}=$ factor de agarre

El factor de distancia horizontal (HM) se calcula utilizando la ecuación 2.:

$$
H M=\frac{25}{H} \quad \text { Ecu. } 2
$$

Donde

$\mathrm{HM}=$ factor de distancia

$\mathrm{H}=$ posición horizontal

EL factor de altura (VM), penaliza los levantamientos cuando se coge las cargas o muy abajo o muy arriba, este factor se le asigna un valor de 1 cuando la carga que se maneja está a 75 cm del suelo (Coronado-Hernandez \& Mateus, 2013). Ver ecuación 3.

$$
V M=[1-0,003(V-75)] \quad \text { Ecu. } 3
$$

Donde

$\mathrm{VM}=$ factor de altura

$\mathrm{V}=$ distancia vertical

Para el factor de desplazamiento vertical (DM) el comité definió un 15\% de disminución del peso cuando se la levante sobre los hombros. Si DM es menor que $25 \mathrm{~cm}$ se asume un valor de 1 . Ver ecuación 4.:

$$
D M=(V 1-V 2) \quad \text { Ecu. } 4
$$

Donde 
$\mathrm{DM}=$ factor de desplazamiento

$\mathrm{V} 1=$ altura inicial

$\mathrm{V} 2=$ altura final

Para calcular el factor de asimetría (AM) se mide el ángulo desde el origen del movimiento considerando el punto medio de una línea imaginaria de los tobillos hasta el final de la posición adoptada en la descarga. El comité escogió un 30\% de disminución cuando el tronco gira $90^{\circ}$ (Carrión-Cevallos et al., 2021). EL valor de AM = 0 cuando el ángulo es superior a $135^{\circ}$. Ver ecuación 5.:

$$
A M=1-(0,0032 A) \quad \text { Ecu. } 5
$$

Donde

$\mathrm{AM}=$ factor de desplazamiento

A= ángulo de descarga

Para el factor de frecuencia (FM) se considera el valor de la frecuencia, la duración del trabajo y el valor de la posición vertical. Ver tabla 1.:

\section{Tabla 1}

Valor de factor de frecuencia

\begin{tabular}{lllllll}
\hline \multirow{2}{*}{$\begin{array}{l}\text { Frecuencia } \\
\text { (elev/min) }\end{array}$} & \multicolumn{3}{c}{ 1 hora o menos } & \multicolumn{2}{c}{ Tiempo de trabajo } & \multicolumn{2}{c}{ De 2 hasta 8 horas } \\
\cline { 2 - 7 } & V 75 & V 75 & V 75 & V 75 & V 75 & V 75 \\
\hline Menos 0,2 & 1,00 & 1,00 & 0,95 & 0,95 & 0,85 & 0,85 \\
0,5 & 0,97 & 0,97 & 0,92 & 0,92 & 0,81 & 0,81 \\
1 & 0,94 & 0,94 & 0,88 & 0,88 & 0,75 & 0,75 \\
2 & 0,91 & 0,91 & 0,84 & 0,84 & 0,65 & 0,65 \\
3 & 0,88 & 0,88 & 0,79 & 0,79 & 0,55 & 0,55 \\
4 & 0,84 & 0,84 & 0,72 & 0,72 & 0,45 & 0,45 \\
5 & 0,8 & 0,8 & 0,6 & 0,6 & 0,35 & 0,35 \\
6 & 0,75 & 0,75 & 0,5 & 0,5 & 0,27 & 0,27 \\
7 & 0,70 & 0,70 & 0,42 & 0,42 & 0,22 & 0,22 \\
8 & 0,60 & 0,60 & 0,35 & 0,35 & 0,18 & 0,18 \\
9 & 0,52 & 0,52 & 0,30 & 0,30 & 0,00 & 0,15 \\
10 & 0,45 & 0,45 & 0,26 & 0,26 & 0,00 & 0,13 \\
11 & 0,41 & 0,41 & 0,00 & 0,23 & 0,00 & 0,00 \\
12 & 0,37 & 0,37 & 0,00 & 0,21 & 0,00 & 0,00 \\
13 & 0,00 & 0,34 & 0,00 & 0,00 & 0,00 & 0,00 \\
14 & 0,00 & 0,31 & 0,00 & 0,00 & 0,00 & 0,00 \\
15 & 0,00 & 0,28 & 0,00 & 0,00 & 0,00 & 0,00 \\
Mas de 15 & 0,00 & 0,00 & 0,00 & 0,00 & 0,00 & 0,00 \\
\hline
\end{tabular}

Nota: Adaptado de (Sánchez \& Vergara, 2019) 
El factor de agarre (CM) se determina de la tabla 2.

\section{Tabla 2}

\section{Valores para el factor de agarre (CM)}

\begin{tabular}{llc}
\hline Tipo de agarre & \multicolumn{1}{c}{$\begin{array}{c}\text { Factor de agarre } \\
(\mathrm{CM})\end{array}$} \\
\cline { 2 - 3 } & V menor 75 & V mayor 75 \\
\hline Bueno & 1,00 & 1,00 \\
Regular & 0,95 & 1,00 \\
Malo & 0,90 & 0,90 \\
\hline
\end{tabular}

Nota: Adaptado de (Yánez, 2019)

\section{RESULTADOS}

Resultados NIOSH inicial, al analizar con el método NIOSH el puesto de trabajo de despacho de pollos se determinaron los siguientes valores iniciales, como se muestra en la tabla 3 .

\section{Tabla 3}

Resultados NIOSH compuesto despacho de pollos

\begin{tabular}{lllll}
\hline ITEM & TAREA 1 & TAREA 2 & TAREA 3 & TAREA 4 \\
\hline Tiempo(h) & 1,5 & 1,5 & 1,5 & 1,5 \\
Peso $(\mathrm{kg})$ & 20 & 25 & 18 & 20 \\
$\mathrm{H}(\mathrm{cm})$ & 25 & 25 & 30 & 30 \\
$\mathrm{~V}(\mathrm{~cm})$ & 70 & 70 & 70 & 75 \\
$\mathrm{D}(\mathrm{cm})$ & 5 & 10 & 5 & 10 \\
$\mathrm{~A}\left({ }^{\circ}\right)$ & 45 & 45 & 0 & 0 \\
$\mathrm{~F}($ lev/min $)$ & 1 & 1 & 2 & 2 \\
Agarre & bueno & regular & malo & malo \\
\hline Nota: H=posición horizontal, $\mathrm{V}=$ distancia vertical, D=desplazamiento de la carga. Adaptado de (Yánez, 2019)
\end{tabular}

Al aplicar las ecuaciones del NIOSH se encontró los siguientes resultados de factores e índices de levantamiento individuales, como se muestra en la tabla 4. 


\section{Tabla 4}

Resultados Factores NIOSH despacho de pollos

\begin{tabular}{lllll}
\hline ITEM & TAREA 1 & TAREA 2 & TAREA 3 & TAREA 4 \\
\hline HM & 1 & 1 & 0,833 & 0,833 \\
VM & 1 & 1 & 1 & 1 \\
DM & 1 & 1 & 1 & 1 \\
AM & 0,856 & 0,856 & 1 & 1 \\
FM & 0,88 & 0,88 & 0,84 & 0,84 \\
CM & 1 & 0,95 & 0.90 & 0,90 \\
LPR & 17,32 & 16,45 & 14,48 & 14,48 \\
IL & 1,15 & 1,51 & 1,24 & 1,28 \\
\hline LPR & Ecuación corregida: & 2,42 \\
COMPUESTO & ILC=IL2(F2)+IL4(F4+F2)-IL4(F2)+IL3(F3+F4+F2)- & \\
& IL3(F4+F2)+IL1(F1+F3+F4+F2)-IL1(F3+F4+F2)* & \\
& ILC=1,51+1,46-1,31+1,74-1,32+2,03-1,69
\end{tabular}

Nota: $\mathrm{HM}=$ Factor de distancia, $\mathrm{VM}=$ Factor de altura, $\mathrm{DM}=$ factor de desplazamiento vertical, $\mathrm{AM}=$ factor de asimetría, $\mathrm{FM}=$ factor de frecuencia, $\mathrm{CM}=$ factor de agarre. * es el resultado del arreglo que recomienda la NTP 477.

Luego de aplicar la ayuda de una persona y bajar las cargas a la mitad se obtuvieron los valores de la tabla 5:

\section{Tabla 5}

Resultados Factores NIOSH despacho de pollos con ayuda

\begin{tabular}{lllll}
\hline ITEM & TAREA 1 & TAREA 2 & TAREA 3 & TAREA 4 \\
\hline Tiempo(h) & 1,5 & 1,5 & 1,5 & 1,5 \\
Peso $(\mathrm{kg})$ & 10 & 12,5 & 9 & 10 \\
$\mathrm{H}(\mathrm{cm})$ & 25 & 25 & 30 & 30 \\
$\mathrm{~V}(\mathrm{~cm})$ & 70 & 70 & 70 & 75 \\
$\mathrm{D}(\mathrm{cm})$ & 5 & 10 & 5 & 10 \\
$\mathrm{~A}\left({ }^{\circ}\right)$ & 0 & 0 & 0 & 0 \\
$\mathrm{~F}($ lev/min $)$ & 1 & 1 & 2 & 2 \\
Agarre & bueno & bueno & bueno & bueno \\
\hline
\end{tabular}

Nota: H=posición horizontal, V=distancia vertical, D=desplazamiento de la carga. Adaptado de (Yánez, 2019)

Al aplicar las ecuaciones del NIOSH se encontró los siguientes resultados de factores e índices de levantamiento individuales, índice compuesto corregido con la ayuda ergonómica: 


\section{Tabla 6}

Resultados Factores NIOSH despacho de pollos con ayuda ergonómica

\begin{tabular}{lllll}
\hline ITEM & TAREA 1 & TAREA 2 & TAREA 3 & TAREA 4 \\
HM & 1 & 1 & 0,833 & 0,833 \\
VM & 1 & 1 & 1 & 1 \\
DM & 1 & 1 & 1 & 1 \\
AM & 1 & 1 & 1 & 1 \\
FM & 0,88 & 0,88 & 0,84 & 0,84 \\
CM & 1 & 1 & 1 & 1 \\
LPR & 20,24 & 20,24 & 19,32 & 19,32 \\
IL & 0,49 & 0,62 & 0,46 & 0,52 \\
\hline LPR & Ecuación corregida: & \\
COMPUESTO & ILC=IL2(F2)+IL4(F4+F2)-IL4(F2)+IL1(F1+F4+F2)- & \\
& IL4(F4+F2)+IL3(F3+F1+F4+F2)-IL1(F1+F4+F2) \\
& ILC=0,43+0,55-0,49+0,60-0,55+0,78-0,72
\end{tabular}

Nota: $\mathrm{HM}=$ Factor de distancia, $\mathrm{VM}=$ Factor de altura, $\mathrm{DM}=$ factor de desplazamiento vertical, $\mathrm{AM}=$ factor de asimetría, FM= factor de frecuencia, $\mathrm{CM}=$ factor de agarre. * es el resultado del arreglo que recomienda la NTP 477.

\section{Discusión}

La implementación de las medidas ergonómicas resulta factible sin embargo involucra el incremento de un puesto de trabajo y por ende el incremento de los costos de operación (Cisneros, 2019). Sin embargo, se debe hacer un seguimiento continuo para contrastar los beneficios, productividad y el ahorro por mejorar el índice de ausentismo laboral.

\section{Conclusiones}

Con el uso de una persona que ayude al levantamiento manual de las cargas se logra bajar un $182 \%$ el índice de levantamiento compuesto garantizando la ejecución de la actividad dentro de los límites recomendados por la legislación ecuatoriana en el Decreto Ejecutivo 2393 Art. 128 Lit. 4.

De los resultados se observa que al poner un puesto espejo mejoran los ángulos de levantamiento y las cargas disminuyen al 50\%. Los índices de levantamiento individual disminuyen significativamente y el índice NIOSH compuesto no supera el valor límite de exposición de 1 . 


\section{Referencias bibliográficas}

Aguirre, Z., \& Ortiz, J. J. R. d. C. d. S. y. D. (2018). Determinantes del riesgo ergonómico y exposición a levantamiento de cargas en trabajadores de una empresa comercializadora de textiles. 3(3), 131-157.

Carrión-Cevallos, M. A., Chávez-Panamito, V. E., Tustón-Torres, I., \& Estuardo-Panchez, M. J. D. d. 1. C. (2021). Análisis del riesgo ergonómico por manipulación manual de cargas en una empresa agrícola. 7(6), 413-428.

Cisneros Rodríguez, M. Á. (2019). Análisis costo-beneficio de la gestión de los riesgos ergonómicos en instituciones de la salud pública. Universidad de Holguín, Facultad de Ciencias Empresariales y Administración ...,

Coronado-Hernandez, J. R., \& Mateus, H. O. J. W.-W. P. o. O. M. (2013). Incorporating ergonomic risks into U-shaped assembly line balancing problem. 4(2), 29-43.

Cuixart, S. N., \& Bravo, M. d. M. C. J. I., Madrid. (1998). NTP 477: Levantamiento manual de cargas: ecuación del NIOSH.

Márquez Gómez, M., Márquez Robledo, M. J. C., \& trabajo. (2015). Factores de riesgo biomecánicos y psicosociales presentes en la industria venezolana de la carne. 17(54), 171-176.

Mora, L. A. (2011). Gestión logística en centros de distribución, bodegas y almacenes-1ra Edición: Ecoe Ediciones.

Muñoz Suárez, A. E. (2019). SISTEMA CONTABLE DE LA EMPRESA EL SEÑOR POLLO DEDICADA A LA ACTIVIDAD DE COMPRA Y VENTA AL POR MENOR DE POLLOS PELADOS EN LA PROVINCIA DEL TUNGURAHUA, CANTÓN AMBATO. Ambato: Universidad Tecnológica Indoamérica

Rodríguez, A. R., Isorni, A. B., \& Yanuzzi, J. P. J. R. N.-S. É. (2010). La Organización Internacional del Trabajo (OIT). Existencia, importancia y trascendencia.

Ruiz, L. J. C. N. d. N. T. (2011). Manipulación Manual de Cargas. Ecuación NIOSH.

Sánchez Ángel, A. M., \& Vergara Cifuentes, C. O. (2019). Evaluación situacional de trabajo en levantamiento de cargas para estibado manual de cajas. Corporación Universitaria Minuto de Dios

Suclli Huaman, E. (2018). Análisis de comercialización de carne de pollo en el mercado de Tancarniyoc, año 2015.

Tobar Cárdenas, T. R. (2018). Exposición a levantamiento manual de cargas y la aparición de lumbalgias en trabajadores del área de impresión de la empresa Sismode”.

Valois Cuero, M. N., \& Rivera Peñuela, J. (2019). Guía práctica para la aplicación de la ecuación de NIOSH en el dolor lumbar. Universidad Santiago de Cali 
Yaguari, P., \& Fabiola, M. (2015). Identificación, evaluación y propuesta de medidas de control de los riesgos ergonómicos biomecánicos por levantamiento de carga en el proceso de estibaje en el área de bodegas de Arca Continental. Universidad Internacional SEK

Yánez Jácome, J. D. (2019). Relación del nivel de riesgo ergonómico según Niosh con los trastornos músculo esqueléticos en estibadores de la Empresa Transerpet SA. PUCE-Quito 


\section{PARA CITAR EL ARTÍCULO INDEXADO.}

Pilatásig Villamarín, S. M., Córdova Suárez, M. A., \& Villacres Cevallos, E. P. (2021). Ayuda ergonómica para atenuar trastornos osteomioarticulares por levantamiento manual de cargas en el despacho de pollos pelados. Anatomía Digital, 4(3.1), 176-186. https://doi.org/10.33262/anatomiadigital.v4i3.1.1919

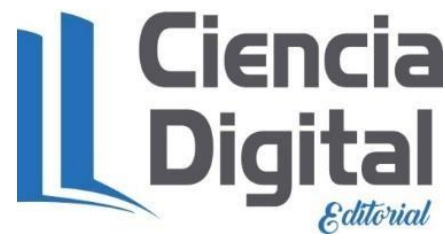

El artículo que se publica es de exclusiva responsabilidad de los autores y no necesariamente reflejan el pensamiento de la Revista Anatomía Digital.

El artículo queda en propiedad de la revista y, por tanto, su publicación parcial y/o total en otro medio tiene que ser autorizado por el director de la Revista Anatomía Digital.
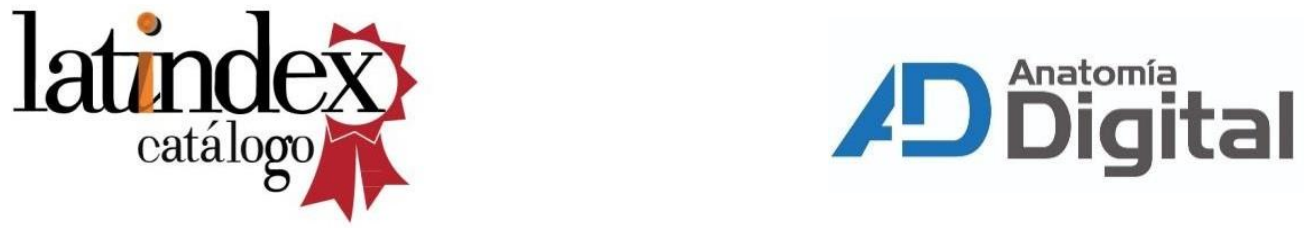